\title{
Neuro-COVID Requires Comprehensive Work-up
}

\author{
Josef Finsterer ${ }^{1}$, Fulvio A Scorza ${ }^{2}$
}

Keywords: Acute demyelinating encephalomyelitis, Coronavirus 2019, Nerve conduction, Polyradiculitis, Severe acute respiratory syndrome coronavirus 2.

Indian Journal of Critical Care Medicine (2021): 10.5005/jp-journals-10071-23926

With interest, we read the article by Mehra et al. about a 13-year old female with the multisystem inflammatory syndrome (MIS) during an infection with severe acute respiratory syndrome coronavirus 2 (SARS-CoV-2), clinically manifesting with multiorgan failure [acute demyelinating encephalomyelitis (ADEM), Guillain-Barre syndrome (GBS), renal insufficiency, myocardial damage, and hepatopathy]. ${ }^{1}$ The patient benefited from ventilatory support, steroids, immunoglobulins, and plasma exchange (PLEX). ${ }^{1}$ It was concluded that MIS can manifest with severe compromise of the central and peripheral nervous system (CNS and PNS). ${ }^{1}$ The study is appealing but raises the following comments and concerns.

The patient was diagnosed with GBS, but the subtype was not specified. According to the electrophysiological data, acute motor axonal neuropathy (AMAN) is the most likely subtype. However, diagnosing GBS according to the Brighton criteria requires cerebrospinal fluid (CSF) investigations and demonstration of a dissociation cyto-albuminique (DCA). ${ }^{2}$ Unfortunately, CSF investigations were not carried out, so the diagnosis of GBS remains questionable.

Since various drugs frequently applied for the treatment of coronavirus 2019 (COVID-19) are neurotoxic, such as daptomycin, linezolide, lopinavir, ritonavir, hydroxychloroquine, cisatracurium, clindamycin, or tocilizumab [Finsterer, submitted], we should know if the patient received any of these compounds in addition to steroids, immunoglobulins, and PLEX. It is also crucial that critical ill neuropathy was excluded as a differential of muscle weakness.

The discrepancy between normal sensory nerve conduction studies (NCSs) and "absent motor responses to pain stimuli" should be explained. According to this statement, the patient rather had acute, motor and sensory, axonal neuropathy (AMSAN) than AMAN.

The same as with GBS is also true for ADEM. ADEM was diagnosed without CSF investigations (usually pleocytosis $>50 / 3$ ). No magnetic resonance imaging of the spinal cord was provided. Grey matter lesions in the basal ganglia and the thalamus were not described. ${ }^{3}$

We do not agree with the notion that simultaneous occurrence of CNS and PNS abnormalities in SARS-CoV-2-infected patients is rare. ${ }^{1}$ If anosmia is regarded as PNS involvement in COVID-19, all COVID-19 patients with anosmia/hyposmia or with dysgeusia/ hypogeusia and CNS disease should be regarded as COVID-19 patients with simultaneous CNS/PNS involvement. Furthermore, if myalgia is regarded as a symptom of muscle involvement in the SARS-CoV-2 infection, all patients with CNS disease and myalgia have simultaneous CNS/PNS involvement. Since ageusia/ anosmia and myalgia are frequent manifestations of COVID-19, simultaneous CNS/PNS involvement in COVID-19 is highly prevalent. Furthermore, Sancho-Saldaña et al. reported a patient with GBS and leptomeningeal enhancement. ${ }^{4}$
${ }^{1}$ Klinik Landstrasse, Messerli Institute, Vienna, Austria

${ }^{2}$ Neuroscience Discipline, Escola Paulista de Medicina/Universidade Federal de São Paulo/(EPM/UNIFESP), São Paulo, Brazil

Corresponding Author: Josef Finsterer, Klinik Landstrasse, Messerli Institute, Vienna, Austria, Phone: +43-1-71165-72085, e-mail: fifigs1@ yahoo.de

How to cite this article: Finsterer J, Scorza FA. Neuro-COVID Requires Comprehensive Work-up. Indian J Crit Care Med 2021;25(8):956-957.

Source of support: Nil

Conflict of interest: None

Recently, it has been recommended to apply infliximab to patients with MIS. Thus, we should know if infliximab was considered as a therapeutic option in the presented patient, and, if applied, if it was beneficial or not.

Missing is the specification of the myocardial damage occurring during the SARS-CoV-2 infection. We should know if the patient was diagnosed with myocarditis, endocarditis, or Takotsubo syndrome (TTS) increasingly recognized as cardiac complications of a SARS-CoV-2 infection. ${ }^{5}$ It is also conceivable that the patient had developed an intraventricular thrombus since SARS-CoV-2 is frequently associated with thrombotic events. The discrepancy between high proBNP and only mild systolic dysfunction should be explained.

Missing are neurological exam and quantification of quadriparesis. Missing are the electroencephalography results. Missing are the NCSs of the phrenic nerve. A limitation is that Table 1 does not provide reference limits.

Overall, this interesting case report has a number of limitations, which should be addressed before conclusions as those presented are drawn. The main limitation is that no CSF investigations were carried out, making the diagnoses of both GBS and ADEM questionable. There is also a need to specify cardiac, gastrointestinal, and mucocutaneous involvement in MIS. Missing information should be provided.

Author contribution: J.F. contributed to design, literature search, discussion, first draft, and critical comments.

Informed consent was obtained.

Ethics approval: The study was approved by the institutional review board.

Data availability: Not applicable.

Consent to participate: Not applicable.

Consent for publication: Not applicable.

(c) Jaypee Brothers Medical Publishers. 2021 Open Access This article is distributed under the terms of the Creative Commons Attribution 4.0 International License (https://creativecommons.org/licenses/by-nc/4.0/), which permits unrestricted use, distribution, and non-commercial reproduction in any medium, provided you give appropriate credit to the original author(s) and the source, provide a link to the Creative Commons license, and indicate if changes were made. The Creative Commons Public Domain Dedication waiver (http://creativecommons.org/publicdomain/zero/1.0/) applies to the data made available in this article, unless otherwise stated. 


\section{OrCID}

Josef Finsterer (1) https://orcid.org/0000-0003-2839-7305

Fulvio A Scorza @i] https://orcid.org/0000-0001-7810-4748

\section{References}

1. Mehra B, Aggarwal V, Kumar P, Kundal M, Gupta D, Kumar A, et al. COVID-19-associated severe multisystem inflammatory syndrome in children with encephalopathy and neuropathy in an adolescent girl with the successful outcome: an unusual presentation. Indian J Crit Care Med 2020;24(12):1276-1278. DOI: 10.5005/ jp-journals-10071-23685.
2. Tan CY, Razali SNO, Goh KJ, Shahrizaila N. Diagnosis of GuillainBarré syndrome and validation of the Brighton criteria in Malaysia. Peripher Nerv Syst 2020;25(3):256-264. DOI: 10.1111/jns.12398.

3. Cole J, Evans E, Mwangi M, Mar S. Acute disseminated encephalomyelitis in children: an updated review based on current diagnostic criteria. Pediatr Neurol 2019;100:26-34. DOI: 10.1016/j. pediatrneurol.2019.06.017.

4. Sancho-Saldaña A, Lambea-Gil Á, Liesa JLC, Caballo MRB, Garay $\mathrm{MH}$, Celada DR, et al. Guillain-Barré syndrome associated with leptomeningeal enhancement following SARS-CoV-2 infection. Clin Med (Lond) 2020;20(4):e93-e94. DOI: 10.7861/clinmed.2020-0213.

5. Finsterer J, Stöllberger C. SARS-CoV-2 triggered Takotsubo in 38 patients. J Med Virol 2020:10.1002/jmv.26581. DOI: 10.1002/jmv.26581. 